\title{
A case of hepatic anisakiasis caused by Pseudoterranova decipiens mimicking metastatic liver cancer
}

\author{
Yasuhiro Murata ${ }^{1 *}$, Katsuhiko Ando ${ }^{2}$, Masanobu Usui ${ }^{1}$, Hiromu Sugiyama ${ }^{3}$, Akinobu Hayashi ${ }^{4}$, Akihiro Tanemura ${ }^{1}$, \\ Hiroyuki Kato', Naohisa Kuriyama', Masashi Kishiwada', Shugo Mizuno ${ }^{1}$, Hiroyuki Sakurai ${ }^{1}$ and Shuji Isaji ${ }^{1}$
}

\begin{abstract}
Background: Anisakid nematodes (Anisakis spp. or Pseudoterranova spp.) usually infect gastric or intestinal walls, while they rarely infect in extra-gastrointestinal sites of human body. Generally, Anisakis spp. larvae are highly infected in fish intermediate hosts, whereas Pseudoterranova spp. larvae are very rarely infected. To the best of our knowledge, there have been no reports which have documented cases of hepatic anisakiasis caused by Pseudoterranova spp. This report describes the first documented case of hepatic anisakiasis due to infection with Pseudoterranova decipiens and clinical features of the hepatic anisakiasis through literature review.

Case presentation: The case was a 28-year-old man with prior history of malignancy who was found to have a hepatic mass mimicking metastatic liver tumor. A new low density area of $20 \mathrm{~mm}$ in diameter in liver segment 7 was found on follow-up CT. With suspicious diagnosis of metastatic liver cancer, laparoscopic partial hepatectomy was performed. A pathological examination revealed no evidence of malignancy, but showed necrotic granuloma with eosinophil infiltration and the presence of a larva with Y-shaped lateral cords, which are specific to anisakid larvae. The type of larva was identified as Pseudoterranova decipiens sensu lato using PCR of DNA purified from a fixed granuloma embedded in paraffin.

Conclusion: The present report is the first to discuss the case of a patient with hepatic anisakiasis caused by Pseudoterranova decipiens. Hepatic anisakiasis is a potential differential diagnosis for hepatic tumors and genetic identification with the PCR method was reliable for obtaining final diagnosis even when the larvae body in the resected specimen collapses with time.
\end{abstract}

Keywords: Anisakis, Extragastrointestinal anisakiasis, Hepatic anisakiasis, Pseudoterranova decipiens, Genetic examination, Metastatic liver cancer

\section{Background}

Anisakiasis is a foodborne disease caused by the accidental ingestion of larval nematodes belonging to the family Anisakidae [1, 2]. Incidence of anisakiasis, at one time relatively common only in East Asia due to the consumption of raw fish, have increased worldwide with the growing popularity of the seafood delicacy [3].

There are two forms, i.e., noninvasive and invasive, of anisakiasis. The noninvasive form is generally asymptomatic

\footnotetext{
*Correspondence: yasumura@clin.medic.mie-u.ac.jp

${ }^{1}$ Department of Hepatobiliary Pancreatic and Transplant surgery, Mie

University Graduate School of Medicine, 2-174 Edobashi, Tsu, Mie 514-8507,

Japan

Full list of author information is available at the end of the article
}

and involves no tissue penetration by the larvae. In case of invasive anisakiasis, anisakid larvae were usually found in the mucosa or submucosa of the gastric and intestinal walls, while they were less commonly detected in extra-gastrointestinal sites of human body [4]. Eosinophilic granuloma is commonly formed around embedded larva in the migrating sites and the histopathologic lesions are changed by the lapse of infection in the chronic invasive anisakiasis [5]. Incidental detection of this lesion is difficult to diagnose and differentiate from recurrence in patients with a prior history of malignancy. This may result in the need for resection, and in some cases the

(c) The Author(s). 2018 Open Access This article is distributed under the terms of the Creative Commons Attribution 4.0 International License (http://creativecommons.org/licenses/by/4.0/), which permits unrestricted use, distribution, and reproduction in any medium, provided you give appropriate credit to the original author(s) and the source, provide a link to the Creative Commons license, and indicate if changes were made. The Creative Commons Public Domain Dedication waiver (http://creativecommons.org/publicdomain/zero/1.0/) applies to the data made available in this article, unless otherwise stated. 
collapsing larvae body may make the definitive diagnosis difficult, even after resection.

Anisakid larvae is commonly classified into two types that have been implicated in human disease: Anisakis (sensu lato) and Pseudoterranova (sensu lato) [6]. Generally, Anisakis spp. larvae are highly infected in fish intermediate hosts, whereas Pseudoterranova spp. larvae are very rarely infected [7, 8]. Anisakis spp. are most often implicated in the invasive type of anisakiasis. In contrast, larvae belonging to the Pseudoterranova spp. are generally noninvasive, and extra-gastrointestinal anisakiasis due to Pseudoterranova spp. is extremely rare. In the present report, we discuss the case of a 28 year-old male with a prior history of malignancy who was diagnosed with an asymptomatic liver tumor mimicking metastatic liver cancer and received a final diagnosis of hepatic anisakiasis caused by Psuedoterranova decipiens through the process of genetic identification. The purpose of the present case study is to describe the first documneted case of hepatic anisakiasis due to infection with Pseudoterranova decipiens and assess clinical features of the hepatic anisakiasis through literature review.

\section{Case presentation}

The patient is a 28 year-old male who had been diagnosed with right testicular cancer. He underwent right high orchidectomy in the department of nephro-urologic surgery in Mie University Hospital in Tsu city, Mie, Japan. The pathological diagnosis was seminoma, pT1N0M0, pStage IA according to the classification established by the Japanese Urological Association [9]. The risk status of tumor was classified as low risk by the International Germ Cell Consensus classification (IGCC) [10]. Thereafter, he received periodic check-ups. Follow-up CT performed at 3 months, 9 months, and 15 months after surgery did not indicate any abnormal findings suggesting recurrence and distant metastasis, but CT performed at 21 months after surgery detected a solitary dumbbell-shaped hypovascular tumor measuring $20 \mathrm{~mm}$ in Segment 7 of the liver (Fig. 1). Abdominal ultrasonography revealed an $18.7 \times 11.4 \mathrm{~mm}$ heterogeneous iso- and hypoechoic mass which displayed an irregular shape and indistinct margin and included hyperechoic spots in segment 7 of the liver, and it did not show flow signal in color doppler mode (Fig. 2). Serum tumor markers, including CEA, CA19-9, AFP, PIVKA-II and hCG, were not found to be elevated. MRI showed a dumbbell-shaped liver tumor in segment 7 which had a low signal intensity on T1-weighted images (T1WI), high signal intensity on T2-weighted images (T2WI), marked signal hyperintensity in diffusion-weighted imaging (DWI), and no signal hypointensity on the ADC map (Fig. 3). PET-CT was performed to confirm the presence of a malignant liver tumor and to search for further metastases in the other organs, but the liver lesion had no specific 18fluoro-deoxyglucose (FDG) uptake compared with normal liver tissue. No other metastasis was detected. Metachronous liver metastasis of testicular cancer was suspected and laparoscopic partial hepatectomy of segment 7 was performed. The resected liver sample included a white nodule of $12 \mathrm{~mm}$ in diameter with a regular border in which a tiny pinhole was present macroscopically (Fig. 4).
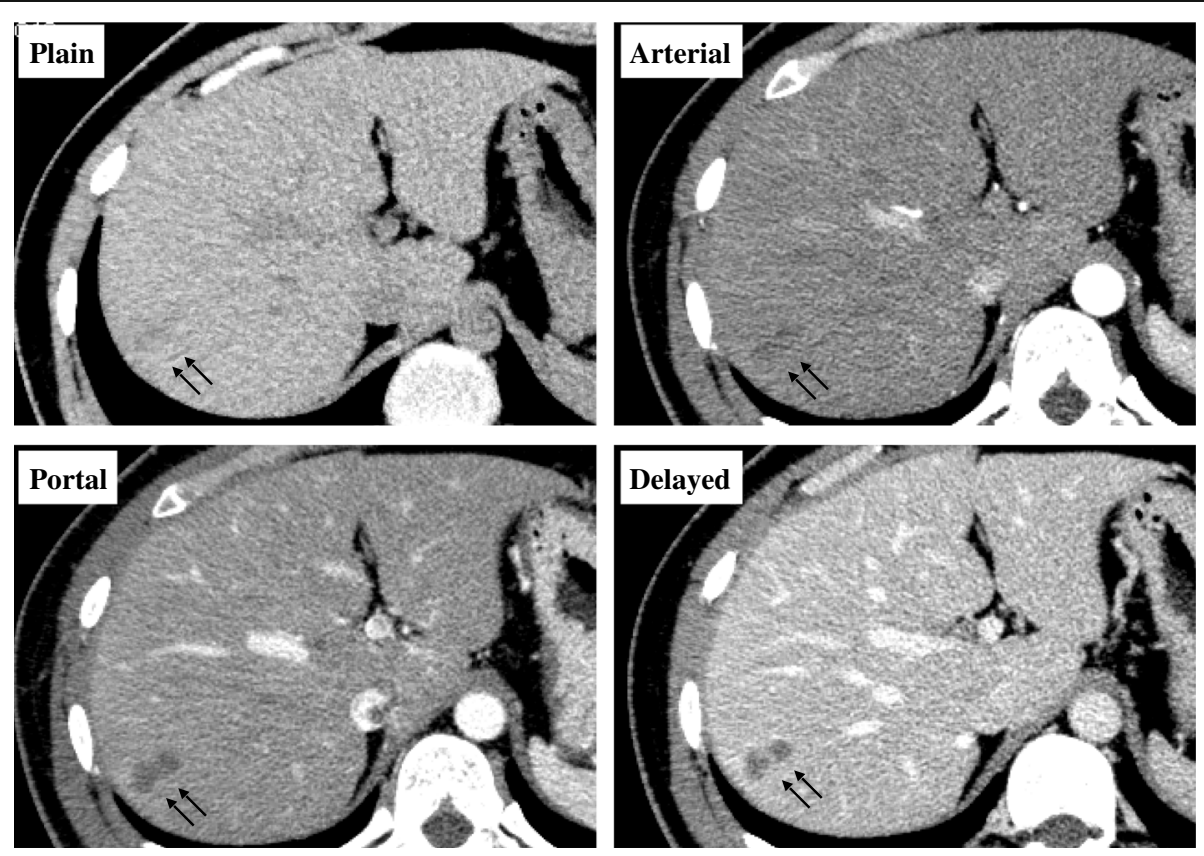

Fig. 1 Axial slice on dynamic enhanced CT before operation. A solitary hypovascular mass detected in segment 7 of the liver (black arrows) 


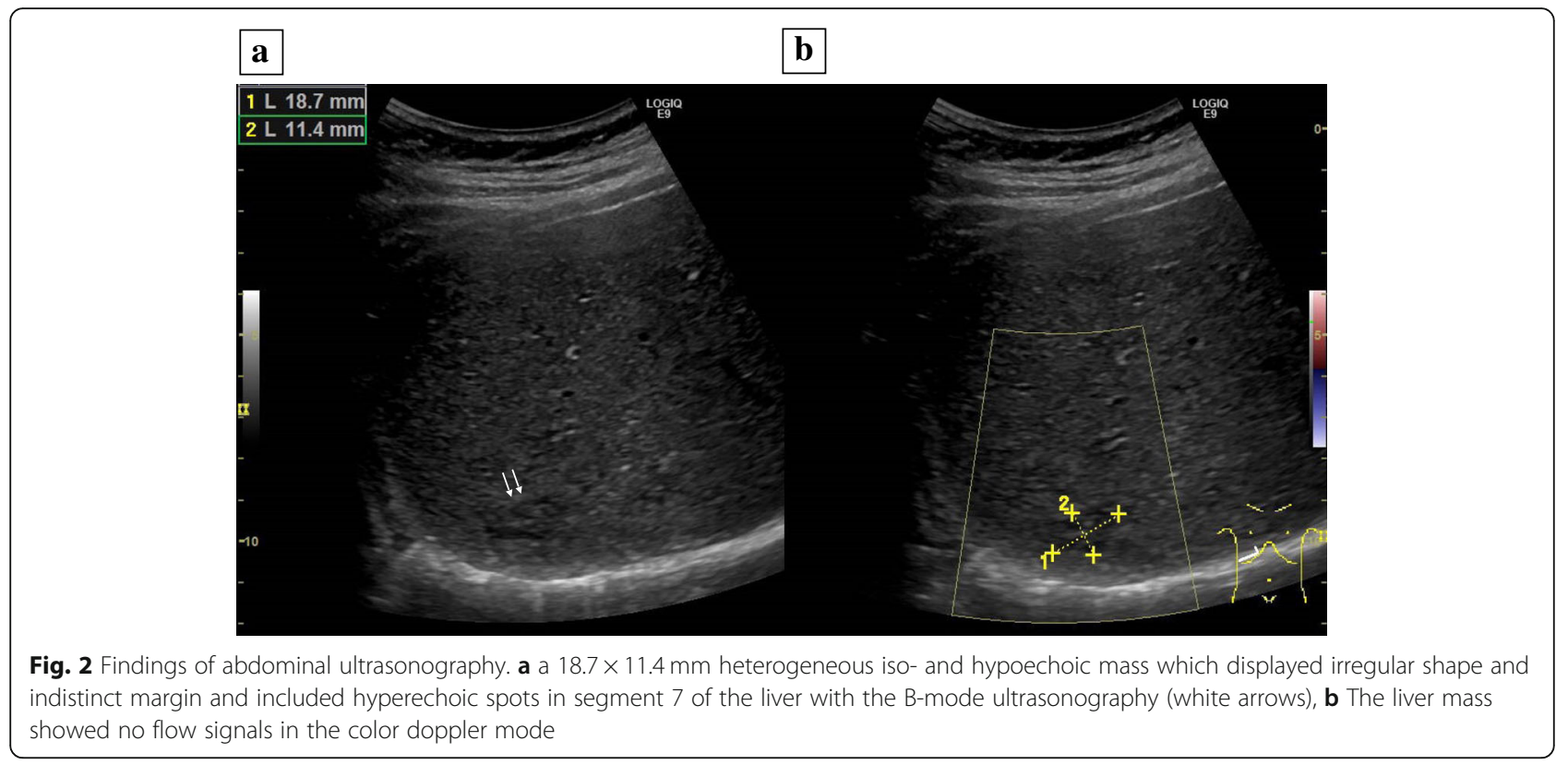

Microscopic examination showed epithelioid granuloma with central necrosis and infiltration of inflammatory cells such as monocytes and eosinophils infiltrated around the granuloma. Within the central necrosis, a small hole showed the presence of exogeneous material. There were no findings consistent with malignancy. The exogenous material displayed a lumen structure which was suspected to be due to larva migrans. A detailed microscopic examination revealed that the larvae had Y-shaped lateral cords, which are specific to anisakid larvae (Fig. 4). However, it was difficult to make a definitive pathological diagnosis, because the larva body had collapsed. Therefore, the slide was sent to the National Institute of Infectious Diseases (NIID) to identify the type of larvae, and genetic examination using PCR method was performed. DNA was extracted from the deparaffinized
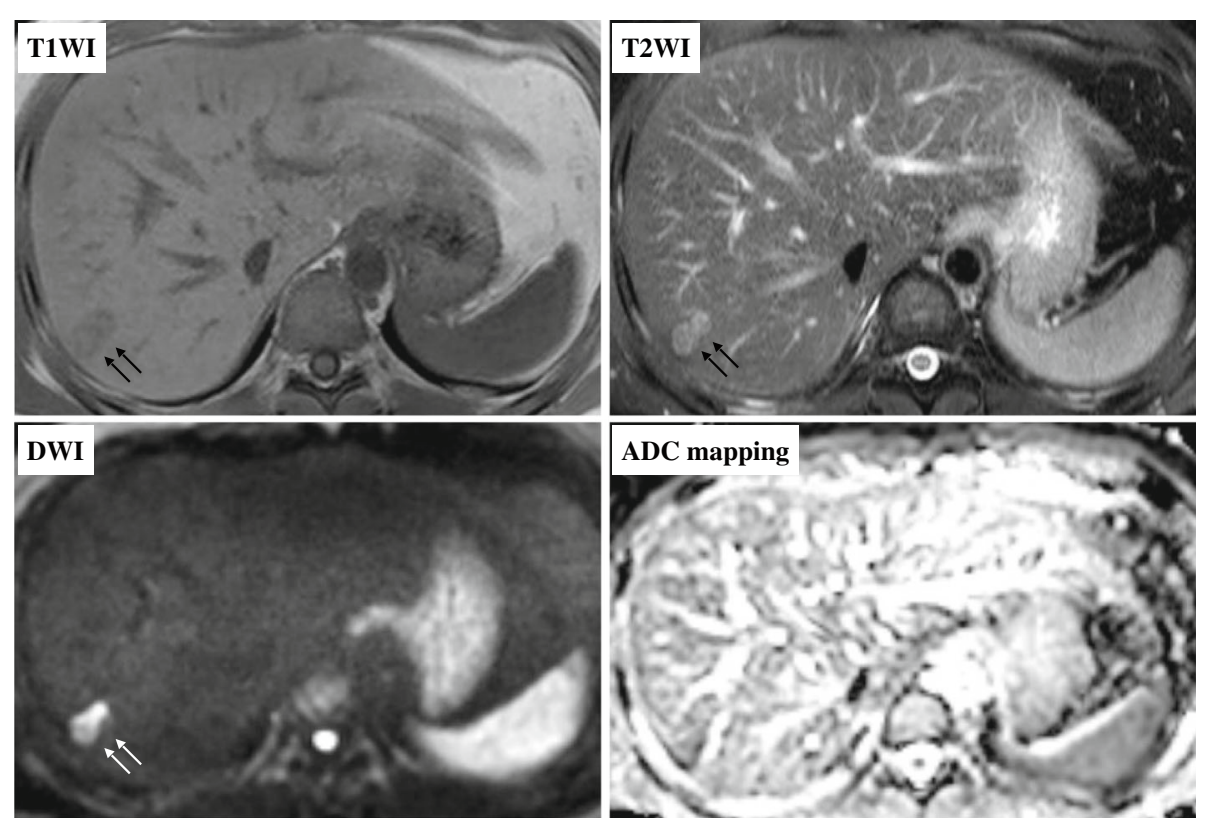

Fig. 3 Axial slice on MRI before operation. The liver mass (dumbbell-shaped) had low signal intensity on T1-weighted images (T1WI), high signal intensity on T2-weighted images (T2Wl), marked signal hyperintensity in diffusion-weighted imaging (DWI), and no signal hypointensity on ADC map 

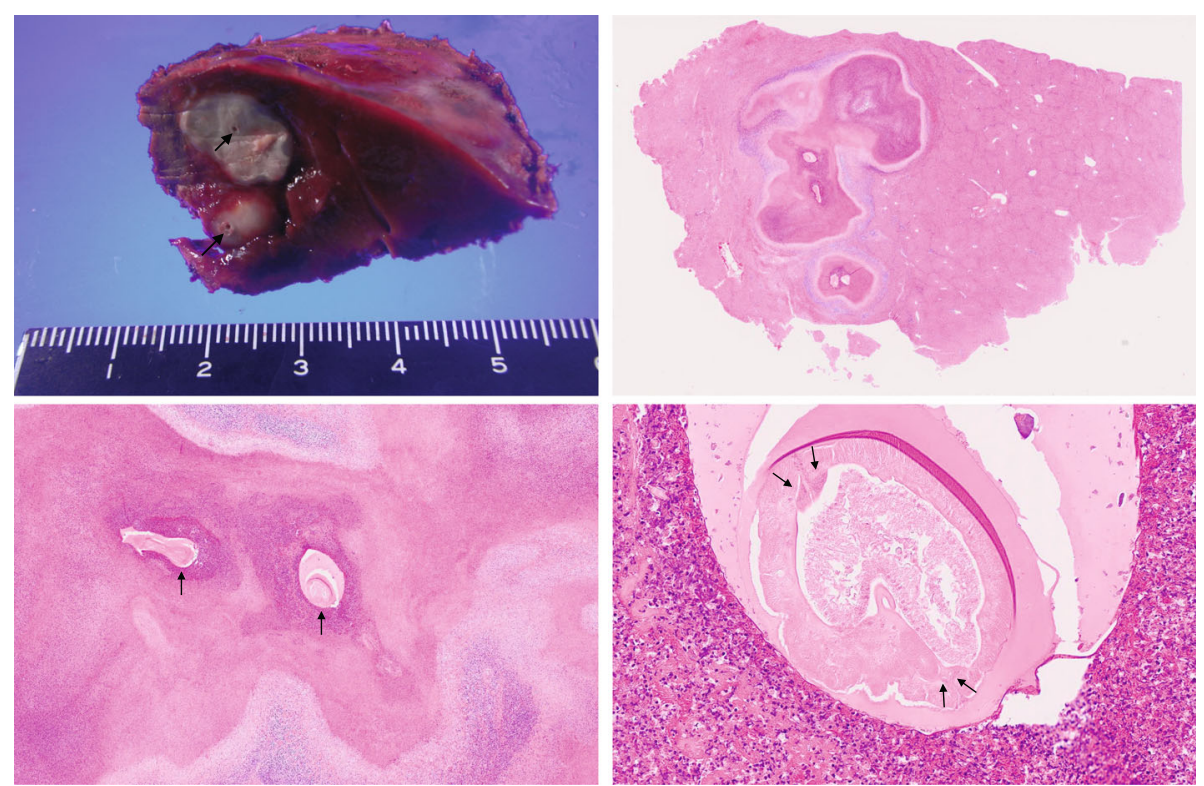

Fig. 4 Macroscopic and microscopic findings of the resected specimen. The resected liver sample included a white nodule of $12 \mathrm{~mm}$ in diameter with a regular border macroscopically. A tiny pinhole was seen within the nodule (upper left, black arrow). Roupe finding showed epithelioid granuloma with central necrosis in the normal liver tissue (upper right, original magnification $\times 0.4$ ). Within the central necrosis, a small hole which bears an exogeneous material was recognizable (lower left, brack arrow, original magnification $\times 2$ ). Axial slice of the larva surrounded by epithelioid granuloma with infiltration of inflammatory cells such as monocytes and eosinophils. The larva body had collapsed, but the specific Y-shaped lateral cord was recognizable (lower right, black arrows, original magnification $\times 20$ )

slide, and the entire internal transcribed spacer (ITS) region (ITS1-28S region) was amplified by PCR using the primers $\mathrm{NC5}$ and $\mathrm{NC} 2$ in the first round. Then nested PCR was performed in the second round to amplify the ITS1-28S region using the following primers that were originally constructed: AniT1F1: 5'-GTTGAACAACGGTG
A CCAATTTGGC-3', and AniT1R1: 5'-GAGTGATCC ACCGCCAAGATTTGTAC-3'. An amplification product of $174 \mathrm{bp}$ was obtained, and its sequence alignment was consistent with that of Pseudoterranova decipiens sensu lato but not with Anisakis simplex sensu stricto (Fig. 5). Finally the liver lesion was pathologically and genetically diagnosed

(i) The primers used for polymerase chain reaction amplification

1 st round for ITS region

NC5: GTAGGTGAACCTGCGGAAGGATCATT (Forward)

NC2: TTAGTTTCTTTTCCTCCGCT (Reverse) (->agcggaggaaaagaaactaa)

2nd round for specific region in ITS (made originally by NIID)

AniT1F1:GTTGAACAACGGTGACCAATTTGGC

AniT1R1:GAGTGATCCACCGCCAAGATTTGTAC (->gtacaatettggcggtggatcactc)

(ii) The sequences of amplified products of control sample (Anisakis simplex) and the present case (Anisakis decipiens)
As
As.
Pd.
Present
1: GTCTACGCCGTATCTAGCTTCTGCCTGGACCGTCAGTTGCGATGAAAGATGCGGAGAAAG 060
Present cas
1 : GTCTACGCCGTATCTAGCTTCTGCCTGGACCGTCGGTAGCGATGAAAGATGCGGAGGAAG 060
As. 61 :TTCCTTTGTTTTGGCTGCTAATCATCATTGATGAGCAGTAGCTTA-AGG-CAGAGTTGA 118
Pd. 61:TTCCTCTGTTTTGGTACGCTAAAGCAGAGTTGAG-CAGACTTAATGA-GCCACGCTTG-- 116
$\begin{array}{ll}\text { Present } & \text { case }\end{array}$
$\begin{array}{lll}\text { As. } & \text { 119: CAGACTTAATGAGCCACGCTAGGTGGCCGCCAAAACCCAAAACACAACCGGTCTATTTGA } & 178 \\ \text { Pd. } & 117:-----154\end{array}$
Present case 117:-----

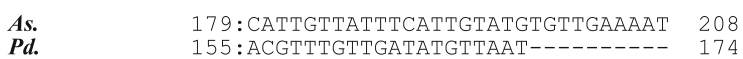
Present case 155: ACGTTTGTTGATATGTTAAT--.------ 174

Fig. 5 Genetic identification of anisakis and sequences obtained. As.: control sample of Anisakis symplex, Pd.: control sample of Pseudoterranova decipiens sensu lato 
as hepatic anisakiasis due to Pseudoterranova decipiens sensu lato. These findings showed the liver lesion was not due to recurrence of testicular cancer. The patient was without recurrence for 2 years and 4 months.

\section{Discussion and conclusions}

This present report is the first to discuss the case of a patient with hepatic anisakiasis caused by Pseudoterranova decipiens. It was difficult to differentiate between a metastatic liver cancer and parasitic granuloma simply by using imaging modalities. Laparoscopic partial hepatectomy for pathological examination and genetic identification of anisakiasis by PCR method were useful for making a final diagnosis even when the larvae body in the resected specimen collapses with time.

Most of extra-gastrointetinal anisakiasis are asymptomatic and detected incidentally [11]. Incidental detection of this lesion is difficult to diagnose and differentiate from recurrence in patients with a prior history of malignancy as in the present case.

Anisakiasis can be categorized into four clinical phenotypes, including gastric, intestinal, extra-gastrointestinal, and allergic [1]. Extra-gastrointestinal anisakiasis accounts for $0.45 \%$ of all cases of anisakis in Japan [12], and hepatic anisakiasis is particularly rare. Only 8 cases including our current case study have been reported in full text articles as case report with detailed medical history, with an English title, based on results from a keyword search of "hepatic anisakiasis" on the PubMed and Japan Medical Abstracts Society websites (Table 1) [11, 13-19]. All cases were reported from Japan. The median age was 59 (range: 28-70), and the subjects, 6 males and 2 females. In most cases, the tumor was located in the surface of liver and presented a low density mass using the contrast enhanced CT scan. The median size of the tumor was 15
(4-20) $\mathrm{mm}$. In general, clinical diagnosis of the hepatic anisakiasis proves to be difficult. All patients underwent hepatectomy with preoperative diagnosis of the liver tumor such as metastatic liver cancer in 5, intrahepatic cholangiocarcinoma in one, and unclassified liver tumor in one. Among these patients, 4 patients obtained a diagnosis of hepatic anisakiasis by histopathological examination and 4 others including our current case study were diagnosed with genetic identification by using of PCR method. In our current case study, a tumor biopsy was considered to be an option for diagnosis that could make surgery unnecessary. However, the specimen obtained with the biopsy was considered unsuitable for definitive diagnosis because of the possibility that the amount of the larvae sample would not be sufficient to perform both a pathological examination and genetic identification. Because our current case study had a prior history of malignancy, we could not exclude the possibility of metastatic liver cancer. It was reported that a granuloma of anisakiasis showed rim enhancement on CT with infusion hepatic arteriography (CTIHA) which appeared similar to the peritumoral enhancement of metastatic cancer, suggesting potential difficulty in making the differential diagnosis between hepatic anisakiasis and metastatic liver cancer on imaging studies [20]. Laparoscopic partial hepatectomy is a minimally invasive surgery, and in terms of the tumor location, the surface area, as in our current case study, is suitable for this procedure. Therefore, laparoscopic partial hepatectomy is an acceptable option for the definitive pathological diagnosis of hepatic anisakiasis and differentiation from metastatic liver cancer. As for the pathological diagnosis of hepatic anisakiasis, the anisakis larvae generally collapses and forms granuloma after migrating into the liver and this may cause difficulty with diagnosis even after resection, as in our case study.

Table 1 Cases of hepatic anisakiasis presenting as a liver tumor including the present case

\begin{tabular}{|c|c|c|c|c|c|c|c|c|c|}
\hline Case & Author & Age & Sex & $\begin{array}{l}\text { Location } \\
\text { (surface or not) }\end{array}$ & Size $(\mathrm{mm})$ & Contrast-enhanced CT & $\begin{array}{l}\text { Preoperative } \\
\text { diagnosis }\end{array}$ & $\begin{array}{l}\text { Definitive } \\
\text { diagnosis }\end{array}$ & Species \\
\hline 1 & Kagei & 51 & M & unknown & 15 & None & Metastatic liver tumor & $\begin{array}{l}\text { Operation } \\
\text { (histopathology) }\end{array}$ & Not assessed \\
\hline 2 & Kawakami & 58 & M & S8 (surface) & 20 & $\begin{array}{l}\text { Low density (delayed } \\
\text { enhancement) }\end{array}$ & Metastatic liver tumor & $\begin{array}{l}\text { Operation } \\
\text { (histopathology) }\end{array}$ & Not assessed \\
\hline 3 & Morita & 59 & $\mathrm{~F}$ & S2 (surface) & 20 & Low density & Liver tumor & $\begin{array}{l}\text { Operation } \\
\text { (histopathology) }\end{array}$ & Not assessed \\
\hline 4 & Ishida & 64 & M & S8 (surface) & 20 & $\begin{array}{l}\text { Low density (peripheral } \\
\text { enhancement) }\end{array}$ & $\begin{array}{l}\text { Intrahepatic } \\
\text { cholangiocarcinoma }\end{array}$ & $\begin{array}{l}\text { Operation } \\
\text { (histopathology) }\end{array}$ & Not assessed \\
\hline 5 & Hayashi & 70 & M & S8 (surface) & 8 & Low density & Metastatic liver tumor & Operation (PCR) & Anisakis pegreffii \\
\hline 6 & Nogami & 44 & $\mathrm{~F}$ & S4 (surface) & 15 & Low density & Metastatic liver tumor & Operation (PCR) & $\begin{array}{l}\text { Anisakis simplex } \\
\text { sensu stricto }\end{array}$ \\
\hline 7 & Sekoguchi & 63 & M & S6 (surface) & 4 & None & None & Operation (PCR) & Anisakis pegreffii \\
\hline 8 & Our case & 28 & M & S7 (surface) & 12 & Low density & Metastatic liver tumor & Operation (PCR) & $\begin{array}{l}\text { Pseudoterranova } \\
\text { decipiens sensu lato }\end{array}$ \\
\hline
\end{tabular}


The PCR methods described here are reliable for obtaining a definitive diagnosis of hepatic anisakiasis in the case studies covered in this paper. In terms of sibling species, which caused hepatic anisakiasis, the Anisakis sensu lato in three cases, including Anisakis pegreffii in two case and Anisakis simplex sense stricto in one, were genetically identified, and the present case is first case in which Pseudoterranova decipiens sensu lato was genetically identified by the PCR method.

Anisakid larvae is commonly classified into two types that have been implicated in human disease: Anisakis (sensu lato) and Pseudoterranova (sensu lato). No studies have been reported about the frequency of species in extragastrointestinal anisakiasis. However, Umehara A. et al. reported that $99 \%$ of all human anisakiasis are due to Anisakis simplex sensu stricto [21].

In general, the invasive capacity of Pseudoterranova spp. larvae is very low relative to that of Anisakis spp. Anisakis spp. are most often implicated in the invasive type of anisakiasis [7, 8, 22]. In contrast, larvae belonging to the Pseudoterranova spp. are generally noninvasive, and hepatic anisakiasis due to Pseudoterranova spp. is particulary rare. In Japan, a case report described ectopic anisakiasis rapidly forming a mass in the left inguinal area in a 5-year old patient caused by Psuedoterranova azarasi. [17]. No reports to date have documented cases of hepatic anisakiasis caused by Pseudoterranova spp. To the best of our knowledge, our current case study is the first to show hepatic anisakiasis caused by Pseudoterranova decipiens, only diagnosed through the process of genetic identification.

The Pseudoterranova decipiens sensu lato, referred to as Pseudoterranova spp., species complex consists of at least 5 sibling species which are genetically but not morphologically distinguishable: $P$. decipiens sensu stricto, $P$. azarasi, P. cattani, P. krabbei, and P. bulbosa [6]. Among these siblings, $P$. decipiens sensu stricto, $P$. azarashi, and $P$. cattani were reported to be the sibling species related to human infection $[6,17,23,24]$. Geographic distribution of the 5 sibling species of the $P$. decipiens complex differs somewhat. $P$. azarasi and $P$. bulbosa are found in the Northwestern Pacific including Japan, $P$. decipiens sensu stricto and P. krabbei in the Northeastern Atlantic, P. decipiens sensu stricto in the Northwestern Atlantic, and $P$. cattani in the Southeastern Pacific waters [25]. The infective larvae of $P$. azarasi were identified from the tissues of various marine fish, including cod, pollack, and smelt and their adult worms live in the intestines of seals and sea lions in the sea Japan [6]. Based on case reports of human infection and geographic distribution, the clinical isolation of our current case study most likely belongs to the $P$. azarasi, although we could not genetically identify which sibling species it belong to.

In conclusion, we experienced a case of hepatic anisakiasis caused by Pseudoterranova decipiens sensu lato. Our results indicate that it is difficult to distinguish hepatic anisakiasis from a metastatic liver cancer on imaging studies for the patients with prior history of malignancy. Genetic identification with the PCR method is reliable for obtaining a final diagnosis even when the larvae body in the resected specimen collapses with time.

\section{Abbreviations \\ ADC: apparent diffusion coefficient; $C T$ : computed tomography; \\ PCR: polymerase chain reaction; PET: positron emission tomography}

\section{Acknowledgements \\ Not Applicable.}

\section{Funding}

This report received no funding.

\section{Availability of data and materials}

The dataset supporting the conclusions of this article is contained within the article.

\section{Authors' contributions}

YM is first author and has performed the entire management of this work which has various parts. Majority of this manuscript has been written by the first author. KA, MU, and SI revised the manuscript. YM, MU, AT, HK, NK, MK, SM, HS, and SI were the doctors in charge for cases and they decided medical options in patient's clinical course, and YM and MU performed surgery. KA and AH diagnosed anisakiasis by pathological examination. YM and KA submitted tissues to NIID for genetic diagnosis after conference with $\mathrm{KA}$ and $\mathrm{AH}$. HS performed genetic examination using PCR method to identify the type of larvae. All authors read and approved the final manuscript.

\section{Ethics approval and consent to participate} Not applicable.

\section{Consent for publication}

Written informed consent was obtained from the patients for publication of this case series and for all accompanying images. A copy of this consent is available for review by the Editor of this journal.

\section{Competing interests}

The authors declare that they have no competing interests.

\section{Publisher's Note}

Springer Nature remains neutral with regard to jurisdictional claims in published maps and institutional affiliations.

\section{Author details}

${ }^{1}$ Department of Hepatobiliary Pancreatic and Transplant surgery, Mie University Graduate School of Medicine, 2-174 Edobashi, Tsu, Mie 514-8507, Japan. ${ }^{2}$ Department of Medical Zoology, Mie University Graduate School of Medicine, Tsu, Japan. ${ }^{3}$ Department of Parasitology, National Institute of Infectious Diseases, Tokyo, Japan. ${ }^{4}$ Department of Pathology, Mie University Graduate School of Medicine, Tsu, Japan.

Received: 28 July 2018 Accepted: 22 November 2018

Published online: 04 December 2018

\section{References}

1. Sakanari JA, McKerrow JH. Anisakiasis. Clin Microbiol Rev. 1989;2(3):278-84.

2. Sohn WM, Chai JY. Anisakiosis (anisakidosis). In: Palmer SR, Soulsby L, Torgerson PR, Brown WG, editors. Oxford textbook of zoonoses. 2nd ed. Oxford: Oxford University Press; 2011. p. 774-86.

3. Baptista-Fernandes T, Rodriques M, Castro I, Paixao P, Pinto-Marques P, Roque L, Belo S, Ferreira PM, Mansinho K, Toscano C. Human gastric hyperinfection by Anisakis simplex: a severe and unusual presentation and a brief review. Int J Infect Dis. 2017;64:38-41. 
4. Chai JY, Darwin Murrell K, Lymbery AJ. Fish-borne parasitic zoonoses: status and issues. Int J Parasitol. 2005;35(11-12):1233-54.

5. Nieuwenhuizen NE. Anisakis - immunology of a foodborne parasitosis. Parasite Immunol. 2016:38(9):548-57.

6. Arizono N, Miura T, Yamada M, Tegoshi T, Onishi K. Human infection with Pseudoterranova azarasi roundworm. Emerg Infect Dis. 2011;17(3):555-6.

7. Arizono N, Yamada M, Tegoshi T, Yoshikawa M. Anisakis simplex sensu stricto and Anisakis pegreffii: biological characteristics and pathogenetic potential in human anisakiasis. Foodborne Pathog Dis. 2012;9(6):517-21.

8. Lim H, Jung BK, Cho J, Yooyen T, Shin EH, Chai JY. Molecular diagnosis of cause of anisakiasis in humans, South Korea. Emerg Infect Dis. 2015;21(2):342-4.

9. Society TJUAatJ. General rule for clinical and pathological studies on testicular tumors. 3rd ed. Tokyo: Kanehara Shuppan; 2005.

10. International Germ Cell Consensus Classification. A prognostic factor-based staging system for metastatic germ cell cancers. International Germ Cell Cancer Collaborative Group. J Clin Oncol. 1997;15(2):594-603.

11. Nogami Y, Fujii-Nishimura Y, Banno K, Suzuki A, Susumu N, Hibi T, Murakami K, Yamada T, Sugiyama H, Morishima Y, et al. Anisakiasis mimics cancer recurrence: two cases of extragastrointestinal anisakiasis suspected to be recurrence of gynecological cancer on PET-CT and molecular biological investigation. BMC Med Imaging. 2016;16:31.

12. Ishikura H, Kikuchi H, Sato N, Ohtani S, Yagi K, Ishikura H, et al. Changing larva migrans caused by anisakidae larvae. Clin Parasitol. 1992;3:70-3.

13. Kagei N, Orikasa H, Hori E, Sannomiya A, Yasumura Y. A case of hepatic anisakiasis with a literal survey for extra-gastrointestinal anisakiasis. Jpn J Parasitol. 1995;44:346-51.

14. Morita M, Soyama A, Takatuki M, Kuroki T, Abe K, Hayashi T, et al. A case of hepatic mass induced by extra-gastrointestinal anisakiasis. J Jpn Surg Assoc. 2013;74:483-7.

15. Ishida M, Sano S, Terada T, Mitsui T, Sudoh Y, Yamaguchi A. A case of hepatic anisakiasis. J Jpn Surg Assoc. 2013;74(9):2557-61.

16. Sekoguchi S, Nagao Y, Takemura K, Yamaguchi K, Dainaka K, Yamada N, Morimoto Y, Isozaki Y, Oyamada H, Ishii H, Kawabata K, Fujita Y, Yamada M. A case of hepatic anisakiasis. Kanzo. 2016;57:577-85.

17. Mitsuboshi A, Yamaguchi H, Ito Y, Mizuno T, Tokoro M, Kasai M. Extragastrointestinal anisakidosis caused by Pseudoterranova azarasi manifesting as strangulated inguinal hernia. Parasitol Int. 2017;66(6):810-2

18. Kawakami S, Ikeda E, Naito M, Yamada M, Tsuji H, Furutani S, Ono K, Otsuka K, Onishi Y, Konishi J, Kunimoto T, Nagasaki S. A case report of hepatc abscess induced by anisakiasis. Okayama Red Cross Hosp J Med. 1995;6(1):92-6.

19. Hayashi R, Sugai K, Yang H, Satoh T, Abo A, Sugai T, Masuda T, Chigusa Y, Sugiyama $\mathrm{H}$. A case of liver anisakiasis found out in the follow-up period after operation of the rectal cancer. Clin Parasitol. 2015;26(1):20-3.

20. Irie T, Takeshita K, Wada Y, Kusano S, Terahata S, Tamai S, Hatsuse K, Aoki H, Sugiura Y. CT evaluation of hepatic tumors: comparison of CT with arterial portography, CT with infusion hepatic arteriography, and simultaneous use of both techniques. AJR Am J Roentgenol. 1995;164(6):1407-12.

21. Umehara A, Kawakami Y, Araki J, Uchida A. Molecular identification of the etiological agent of the human anisakiasis in Japan. Parasitol Int. 2007; 56(3):211-5.

22. Mattiucci S, Paoletti M, Borrini F, Palumbo M, Palmieri RM, Gomes V, Casati A, Nascetti G. First molecular identification of the zoonotic parasite Anisakis pegreffii (Nematoda: Anisakidae) in a paraffin-embedded granuloma taken from a case of human intestinal anisakiasis in Italy. BMC Infect Dis. 2011;11:82.

23. Weitzel T, Sugiyama H, Yamasaki H, Ramirez C, Rosas R, Mercado R. Human infections with Pseudoterranova cattani nematodes, Chile. Emerg Infect Dis. 2015;21(10):1874-5.

24. Brunet J, Pesson B, Royant M, Lemoine JP, Pfaff AW, Abou-Bacar A, Yera H, Frealle E, Dupouy-Camet J, Merino-Espinosa G, et al. Molecular diagnosis of Pseudoterranova decipiens s.s in human, France. BMC Infect Dis. 2017; 17(1):397.

25. Mattiucci S, Nascetti G. Advances and trends in the molecular systematics of anisakid nematodes, with implications for their evolutionary ecology and host-parasite co-evolutionary processes. Adv Parasitol. 2008;66:47-148.

Ready to submit your research? Choose BMC and benefit from:

- fast, convenient online submission

- thorough peer review by experienced researchers in your field

- rapid publication on acceptance

- support for research data, including large and complex data types

- gold Open Access which fosters wider collaboration and increased citations

- maximum visibility for your research: over $100 \mathrm{M}$ website views per year

At BMC, research is always in progress.

Learn more biomedcentral.com/submissions 